\title{
Silicon avalanche photodiode with photon detection efficiency superior to 0.65 electrons/photon in the wavelength range of 114 to $170 \mathrm{~nm}$
}

\author{
Pavel N. Aruev, Victor P. Belik, Vladimir V. Zabrodskii $\odot$,* \\ Andrey V. Nikolaev, and Evgeniy V. Sherstnev \\ Ioffe Institute, St. Petersburg, Russia
}

\begin{abstract}
We have designed a silicon detector based on an avalanche photodiode for detecting vacuum ultraviolet radiation. It was demonstrated that the detector has a photon detection efficiency superior to 0.65 electrons/photon, in the wavelength range from 114 to $170 \mathrm{~nm}$, with an external quantum yield from 49 to 7000 electrons/photon at a reverse-bias voltage from 190 to $315 \mathrm{~V}$, respectively. The detector's active area diameter is $1.5 \mathrm{~mm}$. (C) The Authors. Published by SPIE under a Creative Commons Attribution 4.0 Unported License. Distribution or reproduction of this work in whole or in part requires full attribution of the original publication, including its DOI. [DOI: 10 .1117/1.OE.60.7.077103]
\end{abstract}

Keywords: avalanche photodiode; vacuum ultraviolet; silicon.

Paper 20210235 received Mar. 4, 2021; accepted for publication Jun. 28, 2021; published online Jul. 21, 2021.

\section{Introduction}

Currently, the development of the detectors, which effectively detect vacuum ultraviolet (VUV) is relevant for solving a wide range of scientific problems in the physics of high-temperature plasma, detecting synchrotron radiation, and studying dark matter. ${ }^{1,2}$ This paper is mainly focused on the spectral parameter, which is the most difficult in terms of recording with solid-state detectors. This is the hydrogen Lyman- $\alpha$ emission line at $121.6 \mathrm{~nm}$, having an absorption depth of around $10 \mathrm{~nm}$ for each metal, each semiconductor and more than each second dielectric. ${ }^{3}$

One of the main parameters, characterizing the detector, is a photon detection efficiency $(\varepsilon)$. For non-avalanche silicon photodiodes, the value of $\varepsilon$ was around 1.3 electrons/photon at a wavelength of $160 \mathrm{~nm}$, as demonstrated previously. ${ }^{4}$ Recently, with regard to the same kind of photodiodes, the achieved value of $\varepsilon$ was reported to be at the level of 1.3 to 1.5 electrons/ photon within the 206- to 168 -nm spectral range, respectively. ${ }^{5}$

This paper considers a solid-state avalanche detector design and characteristics. The task of designing a detector with an internal gain and with a photon detection efficiency $(\mathcal{E})$ superior to 0.5 electrons/photon in the full spectrum of VUV radiation, has yet to be achieved. Our previous paper $^{6}$ presented an avalanche silicon precision detector (ASPD). This paper showed that $\varepsilon_{\text {ASPD }}$ (ASPD photon detection efficiency) in the 120- to $170-\mathrm{nm}$ spectral range, was around 0.13 electrons/photon. This value is not a stellar result, compared to the corresponding values of the large area avalanche detectors (LAAPD) presented in Ref. 7. The improved ASPD, designed and studied to increase the $\varepsilon_{\mathrm{ASPD}}$, will be reported upon here.

\section{Improved ASPD}

As already established, there are two dominant loss factors responsible for the value of $\varepsilon$ in a semiconductor detector. These losses are due to the reflection of light, detected in the detector's active area and the collection of losses of photoinduced carriers within the area.

It is notable that in the case of the full spectrum of VUV light, the dielectric, anti-reflection coatings method is unacceptable, since most dielectrics actively absorb VUV light at wavelengths of $<125 \mathrm{~nm},{ }^{3,6}$ which will inevitably lead to a decrease in $\varepsilon$. The previous ASPD $^{6}$ was

*Address all correspondence to Vladimir V. Zabrodskii, sildet@mail.ioffe.ru 


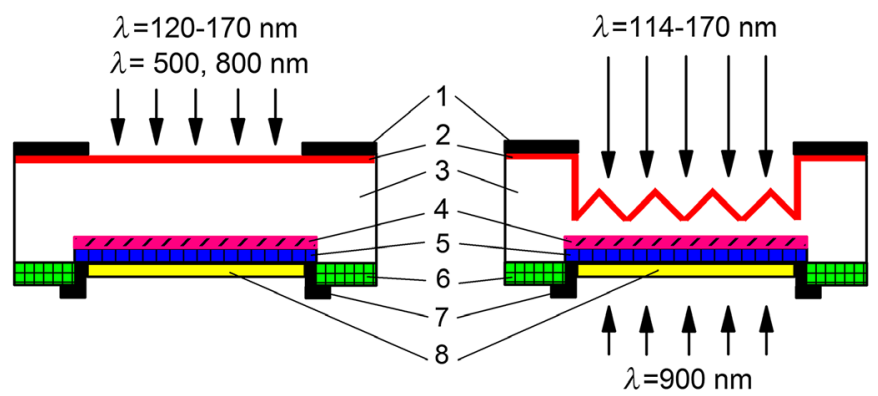

(a)

(b)

Fig. 1 ASPD structures: (a) study; ${ }^{1}$ (b) this study; 1 - aluminum layer, $2-p^{++}$layer, $3-\pi$ region, $4-p$ multiplication layer, $5-n^{++}$layer, 6 - silicon dioxide layer, 7 - aluminum layer, 8 - silicon nitride layer.

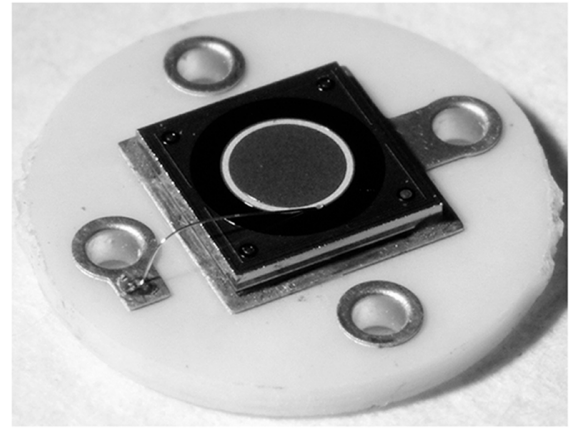

(a)

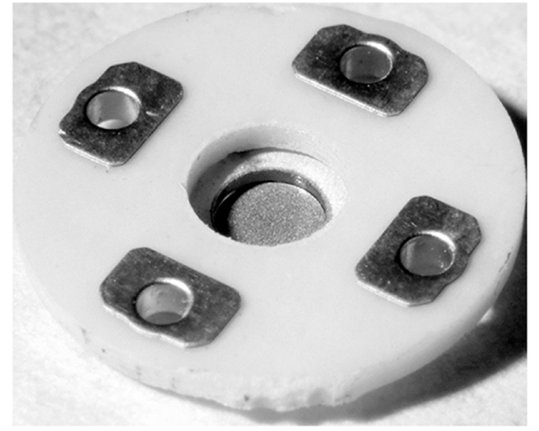

(b)

Fig. 2 Improved ASPD photographs: (a) a front-illuminated mode; (b) a back-illuminated mode.

a $p^{++}-\pi-p-n^{++}$silicon, reach-through, avalanche photodiode. This ASPD was made on a float zone, with [100] orientation from an 8 to $10 \mathrm{kohm}-\mathrm{cm}$ silicon material, with a thickness of $\pi$-region $\sim 320 \mu \mathrm{m}$. The VUV was detected in a back-illuminated mode at normal photon incidence on the flat surface of the isotype $p^{++}-\pi$ junction.

In the case of the ASPD presented, a well-known method of texturing the silicon detector's active area was applied to reduce the reflection losses. Using this method, a pyramid, with a height of around $10 \mu \mathrm{m}$ and apex angles of $54.7 \mathrm{deg}$ could be formed on the silicon surface. A special effort was made to minimize the depth of the isotype $p^{++}-\pi$ junction to reduce any losses associated with the efficiency of collecting photoinduced carriers in the near-surface ASPD active area. The dark current was reduced by halving the $\pi$ region of the improved ASPD, to a value of around $140 \mu \mathrm{m}$. To carry out further research, the metrological base and techniques described in Ref. 6 were used. Figure 1 shows the difference between the structures of the previous ASPD version ${ }^{6}$ and an improved version, ASPD irradiation modes, as well as the difference between the wavelengths used in Ref. 6 and in this paper. To exclude thermal influence while taking measurements, the ASPD's TO-5 package was used, with a high thermal contact to an aluminum thermal capacitor of $1.4 \mathrm{~kJ} / \mathrm{K}$. Considering the amount of power saturation at the ASPD's active area, this would give a stability rate better than $0.49 \mathrm{~K}$ per hour. Figure 2 shows the improved ASPD photographs.

\section{ASPD Multiplication Factor}

The $M_{\mathrm{ASPD}}$ (ASPD multiplication factor) value was found to determine $\varepsilon_{\mathrm{ASPD}}$. The first series of experiments were at determining the $M_{\mathrm{ASPD}}$. To achieve this, we measured the external quantum yield $(\varepsilon)$ of two improved ASPDs in a front-illuminated mode [Fig. 2(a)] at a wavelength of $\lambda=900 \mathrm{~nm}$, therefore, the $\mathrm{Si}_{4} \mathrm{~N}_{4}$ layer (Fig. 1) was irradiated. In relation to these measurements, 
the front-illuminated mode was chosen because it provides more reliable information regarding the thicknesses and composition of the ASPD layers, from the side of the $n^{++}$layer. $\lambda=900 \mathrm{~nm}$ was chosen as it is convenient for estimating radiation absorption in the ASPD structure. There are two reasons for choosing $\lambda=900 \mathrm{~nm}$ to determine $M_{\mathrm{ASPD}}$ in the front-illuminated mode. The first is that estimating the reflection losses is possible at $\lambda=900$, as the surface structure on the $n^{++}$layer is better known from the perspective of manufacturing technology. To estimate the reflection losses, ${ }^{8}$ we took into account the fact that the silicon nitride is $135-\mathrm{nm}$ thick on the $n^{++}$ layer surface. At normal-incidence radiation, the reflection losses amount to around 5\% for $\lambda=900 \mathrm{~nm}$. The second reason is that the absorption depth $\lambda=900 \mathrm{~nm}$ in silicon is approximately $30 \mu \mathrm{m}$. ${ }^{3}$ Given that $135-\mathrm{nm}$ thick $\mathrm{Si}_{3} \mathrm{~N}_{4}$ is transparent for $\lambda=900 \mathrm{~nm}$, it can be argued that the absorption losses in silicon nitride are zero. Considering that the $n^{++}$silicon layer is not thicker than $0.5 \mu \mathrm{m}$, and $\lambda=900 \mathrm{~nm}$ radiation absorption depth is around $30 \mu \mathrm{m}$, the radiation losses in the $n^{++}$layer can also be disregarded. As the base thickness of the studied ASPD is $140 \mu \mathrm{m}$, it is correct to assert that all unreflected radiation for $\lambda=900 \mathrm{~nm}$ will be absorbed in the $\pi$ region of the ASPD and then gained in the multiplication zone.

The $\pi$ region width is much greater than the absorption depth $\lambda=900 \mathrm{~nm}$, therefore, we further assumed that $95 \%$ of the radiation at $\lambda=900 \mathrm{~nm}$, would be absorbed by the $\pi$ region of the ASPDs being studied. This test was carried out using two ASPDs under a reverse-bias voltage of $220 \mathrm{~V}$ in the open air at $23^{\circ} \mathrm{C}$. The measurements were carried out by comparing the photocurrents flowing through the studied ASPDs and through the silicon precision detector (SPD), calibrated ${ }^{9}$ in terms of sensitivity.

The external quantum yield of an ASPD was determined:

$$
\eta_{\mathrm{ASPD}}=\eta_{\mathrm{SPD}} \times I_{\mathrm{ASPD}} / I_{\mathrm{SPD}}
$$

where $I_{\mathrm{ASPD}}$ is the measured photocurrent through an ASPD; $I_{\mathrm{SPD}}$ is the measured photocurrent through the calibrated photodiode and $\varepsilon_{\mathrm{SPD}}$ is the external quantum yield of the calibrated photodiode.

The multiplication factor of an ASPD was determined:

$$
M_{\mathrm{ASPD}}=\eta_{\mathrm{ASPD}} / 0.95
$$

where $\eta_{\text {ASPD }}$ is the calculated external quantum yield of an ASPD and 0.95 is the unreflected radiation factor for $\lambda=900 \mathrm{~nm}$.

Table 1 shows the results for both measuring the external quantum yield $(\eta)$ for two ASPD samples and calculating the $M_{\mathrm{ASPD}}$ at $\lambda=900 \mathrm{~nm}$ under a reverse-bias voltage of $220 \mathrm{~V}$.

\subsection{Photon Detection Efficiency}

The goal of this experiment was to determine $\varepsilon_{\mathrm{ASPD}}$. The $I_{\mathrm{ASPD}}$ photocurrent was measured in the 114- to 170 -nm spectral range in samples, with the $M_{\mathrm{ASPD}}$ determined for a reverse-bias voltage of $220 \mathrm{~V}$, in the back-illuminated mode [Fig. 2(b), irradiated $p^{++}$layer] at $23^{\circ} \mathrm{C}$.

The ASPD photon detection efficiency was determined:

$$
\varepsilon_{\mathrm{ASPD}}=\left(\eta_{\mathrm{SPD}} \times I_{\mathrm{ASPD}}\right) /\left(I_{\mathrm{SPD}} \times M_{\mathrm{ASPD}}\right),
$$

where $\varepsilon_{\mathrm{SPD}}$ is the external quantum yield of the calibrated photodiode; $I_{\mathrm{ASPD}}$ is the measured photocurrent through the ASPD; $I_{\mathrm{SPD}}$ is the measured photocurrent through the calibrated

Table 1 External quantum yield and multiplication factor for the two ASPDs.

\begin{tabular}{lcc}
\hline \hline Sample & $\varepsilon_{\text {ASPD }}$, electrons/photon & $M_{\text {ASPD }}$ \\
\hline No. 9 & 70.6 & 74.3 \\
No. 10 & 120 & 126 \\
\hline \hline
\end{tabular}


Aruev et al.: Silicon avalanche photodiode with photon detection efficiency superior...

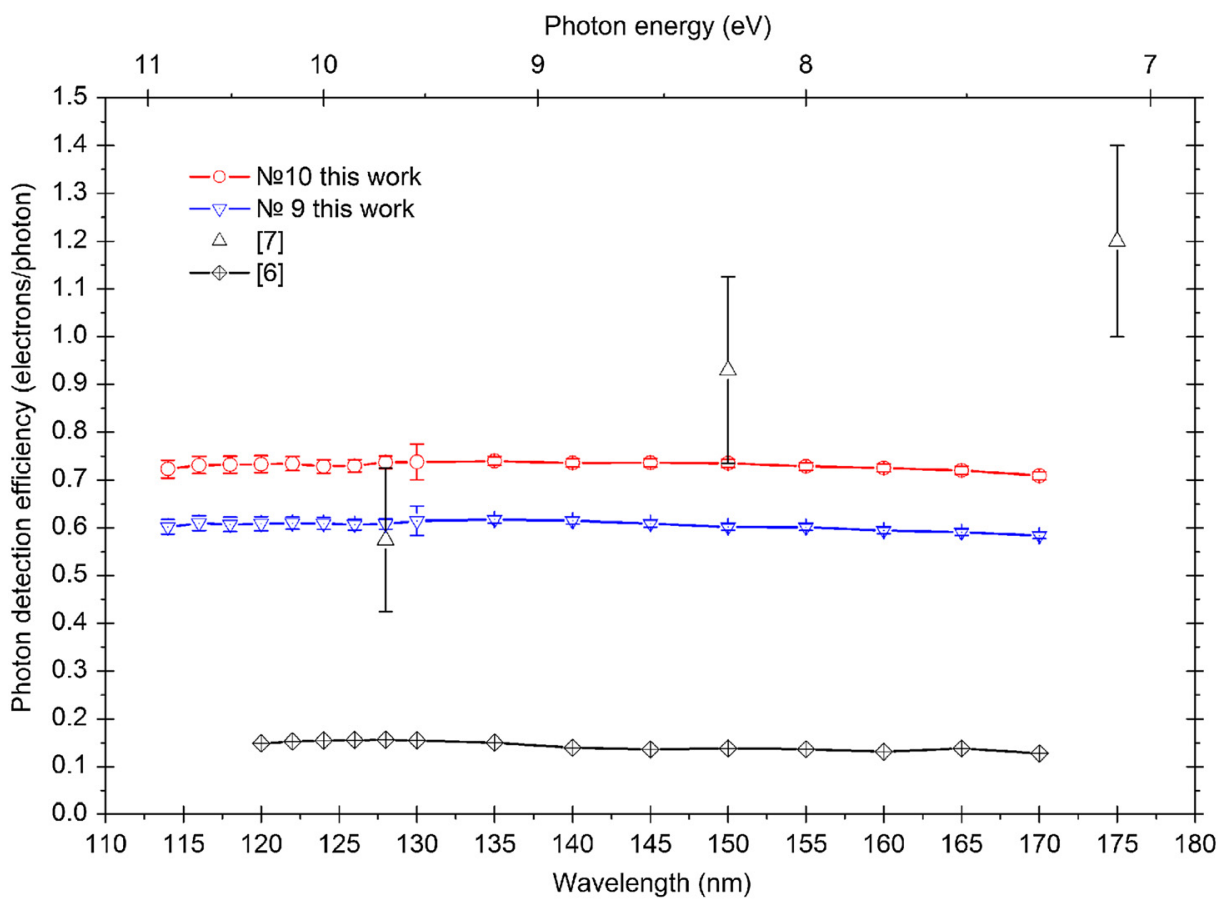

Fig. 3 Photon detection efficiency spectral dependences.

photodiode and $M_{\mathrm{ASPD}}$ is the multiplication factor of an ASPD, calculated as above. Figure 3 shows our $\varepsilon_{\mathrm{ASPD}}$ calculation results and the results from Refs. 6 and 7.

\subsection{Dark Current and External Quantum Yield at $116 \mathrm{~nm}$}

Throughout the final stage, we determined the prospects for using the improved ASPD. The dark current and $\varepsilon_{\mathrm{ASPD}}$ for sample 9 at $23^{\circ} \mathrm{C}$ are shown in Fig. 4.

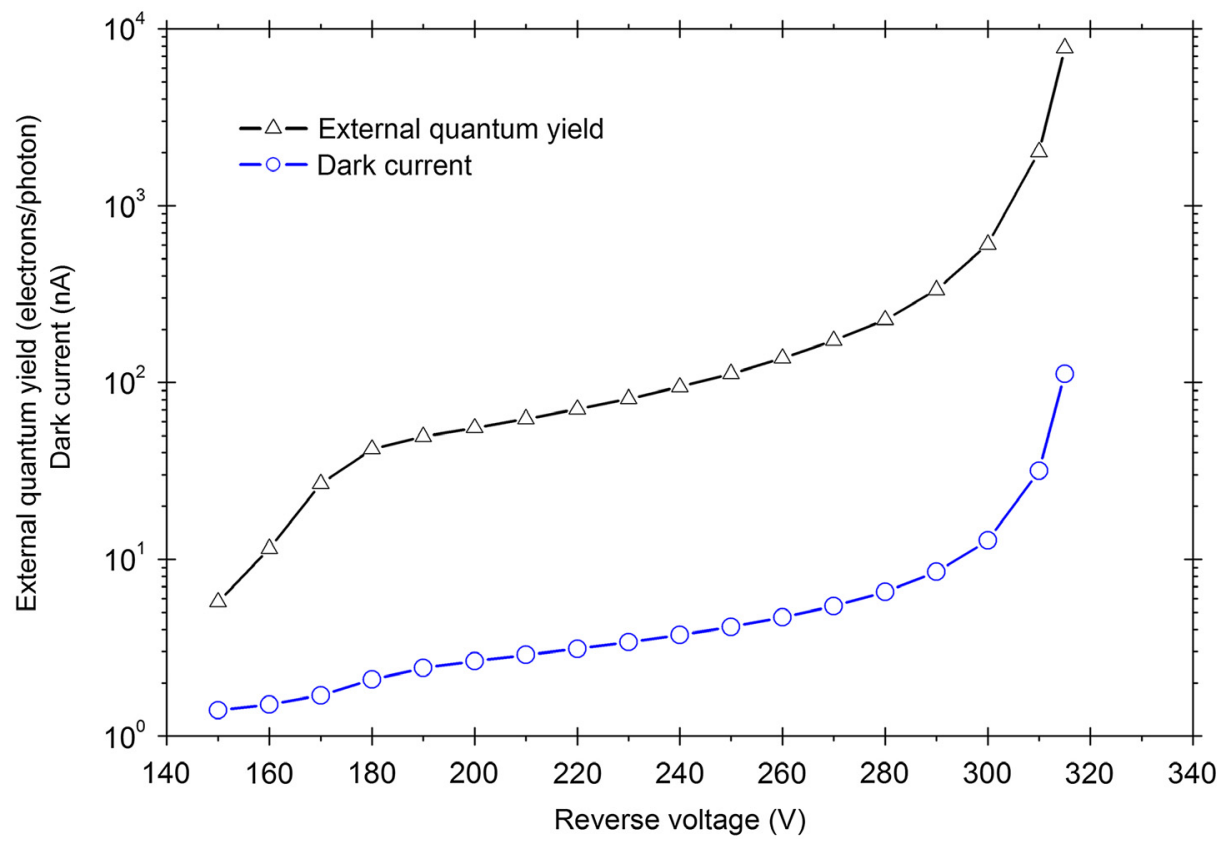

Fig. 4 Improved ASPD external quantum yield at $\lambda=116 \mathrm{~nm}$ and the dark current. 


\section{Discussion}

We investigated experimentally the silicon VUV avalanche detector properties. The improved ASPD proposed, is a combination of a textured surface with a $p^{++}-\pi$ isotype junction on the reverse side of the silicon avalanche photodiode base, with an active area diameter of $1.5 \mathrm{~mm}$. The reduction in reflection losses, due to texturing, provided an increase in $\varepsilon_{\mathrm{ASPD}}$, without the use of dielectric coatings. This made it possible to obtain a quasi-constant $\varepsilon_{\mathrm{ASPD}}$ value in the 114- to 170-nm spectral range, which was an improvement on the detectors presented in Refs. 6 and 7 , and $\varepsilon_{\mathrm{ASPD}}$ was more than five times higher than that recorded using the first ASPD. ${ }^{6} \mathrm{~A}$ set of quasi-constant spectral dependences and $\varepsilon_{\mathrm{ASPD}}$ superior to 0.65 electrons/photon, is very convenient from a metrological point of view. $\varepsilon_{\mathrm{ASPD}}<1$ electrons/photon may indicate that increasing the effective detector dead layer is essential, due to an abnormal VUV incidence angle in the case of a textured active area surface.

ASPDs have an active region structure formed by the same method as in SPDs, i.e., with a dead layer thickness of around $8 \mathrm{~nm} .{ }^{10}$ Hence, the possibility of using an ASPD in the full spectrum of VUV radiation should be expected, and not only when measuring its studied element in the 114-170-nm spectral range, which is the most difficult from a VUV detection point of view. ${ }^{6}$ This expectation is based on the spectral dependence of $\varepsilon$ on such a calibrated SPD $p^{++}-n$ photodiode $^{9,11,12}$ [Fig. 5(a)], which has a textured active region with a $p^{++}$layer. As can be seen from Fig. 5(a), in the case of a calibrated SPD and an improved ASPD, we can consider the correlation of their spectral dependences, i.e., the $\varepsilon$, with the spectral dependences of the absorption depths of ultraviolet photons in boron ${ }^{13}$ and silicon. ${ }^{3}$ An increase in the $\varepsilon$ values of the calibrated SPD for wavelengths $<125 \mathrm{~nm}$, is associated with a significant increase in the absorption depth in silicon, as well as an increase in the contribution of the impact ionization effect in silicon, due to an increase in the energy of the quanta being detected. ${ }^{14}$ It should be noted that the active region of the $p^{++}$layer, used in these detectors, can be characterized as a solid solution of boron and silicon, coated with an amorphous boron silicide layer $\sim 8$-nm thick. ${ }^{10}$ The lower $\varepsilon$ value in relation to the calibrated SPD (relative to the improved ASPD) is associated with the increased collection losses of photoinduced carriers within the active area. These losses are due to the greater $p^{++}-n$ junction depth and the higher dead layer thickness in the case of the calibrated SPD, than in the isotype $p^{++}-\pi$ junction of the improved ASPD structure.

In the case of LAAPD, ${ }^{7}$ as the wavelength decreases from 175 to $128 \mathrm{~nm}$, a twofold decrease in the value of $\varepsilon$ is observed [Fig. 5(b)]. This decrease is most likely associated with an increase in absorption in the dielectric coating of the active region. The interference factor should not be ruled out, as can be seen in Ref. 4 using $\mathrm{SiO}_{2}$ as an example. In the case of improved ASPD, interference is not a first-order factor, due to the limited thickness of the amorphous layer (no more than $8 \mathrm{~nm}$ ). In addition, the spectral dependences of the ultraviolet quanta absorption depth in silicon and boron [Fig. 5(a)], do not demonstrate sharp decreases in values in the range of 50 to $250 \mathrm{~nm}$, by contrast with the similar characteristics of dielectrics [Fig. 5(b)]. We could not find any data on the ultraviolet quanta absorption depth for boron silicides in the referenced literature.

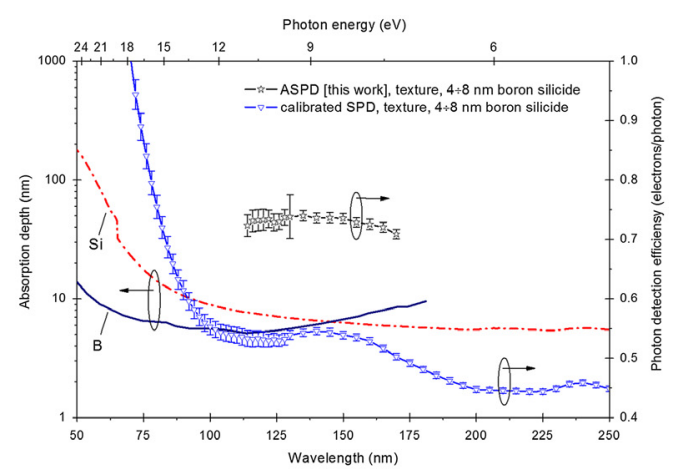

(a)

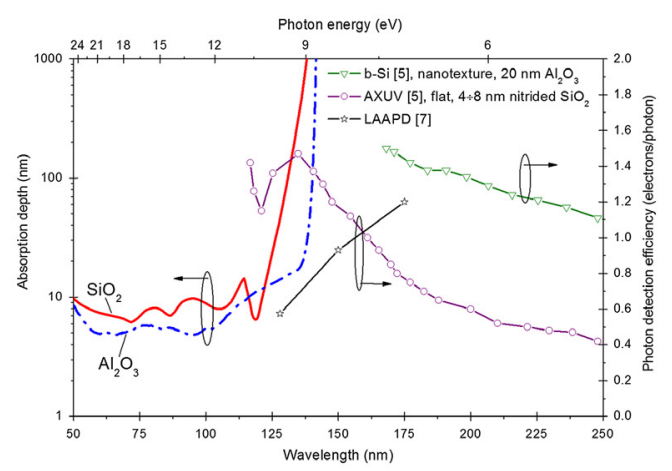

(b)

Fig. 5 Spectral dependences of the ultraviolet quanta absorption depth and the photon detection efficiency. 
Figure 5(b) shows the spectral dependences of $\varepsilon$ for non-avalanche silicon photodiodes, made using black-silicon (b-Si) technology ${ }^{15}$ and well-known, non-avalanche AXUV photodiodes. ${ }^{16}$ The data for b-Si and AXUV photodiodes were taken from Ref. 5. As one can see from the referenced literature, the active region of $\mathrm{b}-\mathrm{Si}$ photodiodes is covered with a $20-\mathrm{nm}$ thick $\mathrm{Al}_{2} \mathrm{O}_{3}$ layer ${ }^{15}$ and the AXUV active region is covered with a 4- to 8-nm thick nitrided $\mathrm{SiO}_{2}$ layer. ${ }^{16}$ Figure 5(b) also demonstrates the spectral dependences of the ultraviolet quanta absorption depth for $\mathrm{SiO}_{2}{ }^{3}$ and for $\mathrm{Al}_{2} \mathrm{O}_{3} \cdot{ }^{17}$ As can be seen from Fig. 5(b), the spectral dependence of $\varepsilon$ in the case of AXUV exhibits a pronounced dip at a wavelength of $\sim 121 \mathrm{~nm}$, which correlates with the local minimum of the absorption depth of VUV quanta for $\mathrm{SiO}_{2}$. It follows from this that the nitrided $\mathrm{SiO}_{2}$, used in AXUV, is similar in terms of its optical properties to $\mathrm{SiO}_{2}$. There is every reason to believe that the decrease in AXUV by $\sim 25 \%$ in the region of $121 \mathrm{~nm}$, is associated with a sharp increase in losses in the active region of AXUV, due to VUV absorption in the nitrided $\mathrm{SiO}_{2}$. A similar effect should be expected in the case of b-Si photodiodes. A decrease in the value of $\varepsilon$ for b-Si photodiodes by tens of percent, will be due to an increase in the loss of VUV quanta as a result of the absorption in the $\mathrm{Al}_{2} \mathrm{O}_{3}$ layer in relation to wavelengths less than $130 \mathrm{~nm}$. Moreover, b-Si photodiodes will exhibit higher losses due to the greater thickness of $\mathrm{Al}_{2} \mathrm{O}_{3}$ than that of nitrided $\mathrm{SiO}_{2}$, in the case of AXUV. The presence of nanotexture ${ }^{15}$ will be an additional negative factor that increases the loss of VUV radiation in $\mathrm{Al}_{2} \mathrm{O}_{3}$ for b-Si photodiodes. Nanotexture forms an angle of radiation incidence on the $\mathrm{Al}_{2} \mathrm{O}_{3}$ surface of $\sim 20 \mathrm{deg}$. The effective thickness of the $\mathrm{Al}_{2} \mathrm{O}_{3}$ layer will be more than $20 \mathrm{~nm}$ for the radiation incidence, usually associated with the active region of the b-Si photodiodes.

It should be noted that in addition to the aforementioned significant parameter, $\varepsilon$, there are other equally important properties of photodiodes, for example, the resistance of their active region to ozone cleaning of the surface from carbon contamination, ${ }^{18}$ as well as the resistance of the photodiodes' active region to VUV radiation. ${ }^{4,16,19}$ VUV radiation induces an additional positive charge in the irradiated zone of the photodiode active region, changing the initial surface charge. A change in the surface charge of the active region of the detecting zone of the photodiode, leads to a change in the losses associated with the collection of minority charge carriers. As a result, changing these losses decreases or increases the value of $\varepsilon$. A change in $\varepsilon$ at the primary stage can lead to an increase in its value, ${ }^{4}$ but ultimately leads to its decrease and degradation of the photodiode. ${ }^{4,20,21}$ In light of the above, it would be extremely interesting to obtain the results of the radiation hardness tests for b-Si photodiodes at wavelengths of 121.6 and $193 \mathrm{~nm}$.

The measurements were carried out by direct comparison with an absolutely calibrated photodiode using a gas-discharge lamp and a monochromator. This resulted in fewer errors than in Ref. 7. The measurement error was determined by the calibration ${ }^{9}$ accuracy of the reference SPD photodiode ( $3 \%$ to $6 \%$ ) at all wavelengths, except for $\lambda=130 \mathrm{~nm}$, where a low flux density from a gas-discharge lamp was detected by a calibrated photodiode, with an accuracy of $10 \%$.

It should be noted that despite the high ASPD external quantum yield, AXUV detectors ${ }^{16}$ or SPDs are more suitable for VUV absolute measurements. In these detectors, there is no temperature-dependent avalanche multiplication of photoinduced carriers. In addition, the silicon detectors, based on avalanche multiplication, are limited by the maximum photocurrent of $\sim 0.2 \mathrm{~mA}$ (for $M_{\mathrm{ASPD}} \sim 100$ ), due to the danger of irreversible thermal breakdown.

There is no such limitation for detectors without avalanche multiplication. Nevertheless, detectors with an internal gain, make it possible to solve the problems of recording the dynamics of VUV radiation, with a flux density 100 or more times lower than detectors without an internal gain.

\section{Conclusion}

This paper has presented a new silicon detector for VUV radiation detection, with a photon detection efficiency superior to 0.65 electrons/photon in the full 114- to 170-nm spectral range; this detector operates at modes with an external quantum yield between 49 and 7000 electrons/ photon at a reverse-bias voltage from 190 to $315 \mathrm{~V}$, respectively. Further investigations will be aimed at studying the temperature dependences of the photon detection efficiency in the VUV range, as well as the noise characteristics of the presented avalanche photodiode. 


\section{Acknowledgments}

We are grateful to our colleagues from the Ioffe Physical Technical Institute, namely N.V. Zabrodskaya, M.S. Lazeeva, M.V. Drozdova, and V. I. Marshalova, for their help in preparing photodiodes, and we would like to thank M.E. Levinshtein for reading the manuscripts and making valuable remarks.

\section{References}

1. P. Agnes et al., "Direct search for dark matter with DarkSide," J. Phys. Conf. Ser. 650, 012006 (2015)

2. W. Zheng, L. Jia, and F. Huang, "Vacuum-ultraviolet photon detections," iScience 23(6), 101145 (2020).

3. E. D. Palik, Ed., Handbooks of Optical Constants of Solids, Vol. 1, Academic Press, Cambridge, Massachusetts (1998).

4. L. R. Canfield et al.,"Absolute silicon photodiodes for $160 \mathrm{~nm}$ to $254 \mathrm{~nm}$ photons,' Metrologia 35, 329-334 (1998).

5. T. Tsang et al., "Quantum efficiency of black silicon photodiodes at VUV wavelengths," Opt. Express 28, 13299-13309 (2020).

6. P. N. Aruev et al., "Quantum yield of a silicon avalanche photodiode in the wavelength range of 120-170 nm,” Tech. Phys. 65(8), 1333-1339 (2020).

7. L. R. Chandrasekharan, M. Messina, and A. Rubbia, "Detection of noble gas scintillation light with large area avalanche photodiodes (LAAPDs)," Nucl. Instrum. Methods Phys. Res. Sect. A 567(1), 45 (2006).

8. M. Born and E. Wolf, Principles of Optics, 7th ed., Cambridge University Press, Cambridge (1999).

9. A. Gottwald et al., "Ultraviolet and vacuum-ultraviolet detector-based radiometry at the Metrology Light Source,” Meas. Sci. Technol. 21, 125101 (2010).

10. V. Zabrodskii et al, "SiPM prototype for direct VUV registration," Nucl. Instrum. Methods Phys. Res. Sect. A 787, 348 (2015).

11. F. Scholze, R. Klein, and R. Müller, "Characterization of detectors for extreme UV radiation," Metrologia 43, S6-S10 (2006).

12. P. N. Aruev et al., "The architecture of a $32 \times 32$ hybrid matrix format high-speed detector for spectral range vacuum ultraviolet-hard x-rays," Instrum. Exp. Tech. 64(1), 93-96 (2021).

13. M. Fernández-Perea et al, "Optical constants of electron-beam evaporated boron films in the 6.8-900 eV photon energy range," J. Opt. Soc. Am. A 24, 3800-3807 (2007).

14. S. Kolodinski et al., "Quantum efficiencies exceeding unity due to impact ionization in silicon solar cells," Appl Phys Lett 63, 2405 (1993).

15. M. Garin et al., "Black-silicon ultraviolet photodiodes achieve external quantum efficiency above 130\%," Phys. Rev. Lett. 125, 117702 (2020).

16. R. Korde, J. S. Cable, and L. R. Canfield. "One gigarad passivating nitrided oxides for 100\% internal quantum efficiency silicon photodiodes," IEEE Trans. Nucl. Sci. 40, 1655 (1993).

17. R. H. French, H. Mullejans, and D. J. Jones, "Optical properties of aluminum oxide: determined from vacuum ultraviolet and electron energy-loss spectroscopies," J. Am. Ceram. Soc. 81(10), 2549-2557 (1998).

18. F. Scholze, R. Klein, and T. Bock, "Irradiation stability of silicon photodiodes for extremeultraviolet radiation," Appl. Opt. 42(28), 5621 (2003).

19. V.V. Zabrodsky et al., "A study of vacuum ultraviolet stability of silicon photodiodes," Tech. Phys. Lett. 38(9), 812-815 (2012).

20. P. N. Aruev et al., "Characterization of spatial homogeneity of sensitivity and radiation resistance of semiconductor detectors in the soft x-ray range," Nucl. Instrum. Methods Phys. Res. A 603 58-61 (2009).

21. V. V. Zabrodsky et al., "Study of EUV and x-ray radiation hardness of silicon photodiodes," Proc. SPIE 8777, 87770R (2013).

Biographies of the authors are not available. 